\title{
Altered Treg and cytokine responses in RSV-infected infants
}

\author{
Allison F. Christiaansen', Muhammad A. Syed ${ }^{2}$, Patrick P. Ten Eyck³, Stacey M. Hartwig', Lakshmi Durairaj', Sameer S. Kamath² \\ and Steven M. Varga ${ }^{1,5,6}$
}

BACKGROUND: Respiratory syncytial virus (RSV) is the leading cause of bronchiolitis and pneumonia in children under $1 \mathrm{y}$ of age in the USA. The host immune response is believed to contribute to RSV-induced disease. We hypothesize that severe RSV infection in infants is mediated by insufficient regulation of the host immune response of regulatory $T$ cells (Tregs) resulting in immunopathology.

METHODS: Blood and nasal aspirates from 23 RSV-infected and 17 control infants under 1 y of age were collected. Treg frequencies were determined by flow cytometry from peripheral blood mononuclear cells. Analysis of 24 cytokines was measured by multiplex assay on nasal aspirates.

RESULTS: We demonstrate that the frequency of activated Tregs is significantly reduced in the peripheral blood of RSV-infected infants compared with age-matched controls. Surprisingly, T helper (Th)17 related cytokines including interleukin (IL)-1 $\beta$, IL-17A, and IL-23 were associated with a reduction in clinical symptoms of respiratory distress. In addition, the amount of IL-33 protein in nasal washes, a cytokine important in maintaining Treg homeostasis in mucosal tissues, was decreased in RSV-infected children.

CONCLUSION: These results suggest that decreased Treg numbers and an inability to properly control the host inflammatory response results in severe RSV infection.

$\mathbf{R}$ espiratory syncytial virus (RSV) is the leading cause of lower respiratory tract infections in young children worldwide (1). The majority of children become infected with RSV during the first year of life, with almost all children exposed to RSV by the age of 2 y (2). Each year an estimated 33.8 million annual RSV infections occur resulting in 3-4 million hospitalizations and 66,000-199,000 deaths worldwide in children under $5 \mathrm{y}$ of age (1). While a number of risk factors have been identified that are associated with the chance of severe RSV infection, the majority of RSV hospitalizations occur in previously healthy children (3). Currently, the only approved RSVspecific therapy is prophylactic treatment with palivizumab $(4,5)$. However, preventative treatment with palivizumab is restricted to high-risk infants and therefore, there is currently no approved methods to prevent severe RSV-induced disease in otherwise healthy children (6). The development of an RSV vaccine is needed to reduce the global disease burden and mortality observed from RSV infection. Thus, an increased understanding of the host immune response to severe RSV infection in infants would help facilitate the development of an effective vaccine and/or therapeutic intervention.

The host immune response is thought to contribute to the severity of RSV-induced disease in both humans (7) and animal models $(8,9)$. Conflicting reports exist as to the specific role of the T cell response in RSV-induced disease. Both CD4 and CD8 T cells have been detected in the airways of RSVinfected infants $(7,10,11)$. However, $\mathrm{T}$ cells are present at a much lower frequency than other inflammatory cells such as neutrophils and monocytes $(7,10)$. Notably, many CD4 Tcell-specific cytokines have been detected and implicated in human RSV-induced disease (12-14). In mice, RSV-induced disease has been shown to be mediated by an improper skewing of CD4 T cell subsets $(9,15,16)$. T helper (Th) 1 polarized CD4 T cells that produce IFN- $\gamma$ and TNF- $\alpha$ contribute to the clearance of viral infections, however, RSV infection often induces both Th2 and Th17 responses (16-18). Thus, regulation and induction of a proper immune response is vital to the prevention of RSV-induced disease (19). Regulatory T cells (Tregs) have been shown to play a critical role in preventing disease in RSV-infected mice (20-23). Tregs have been shown to prevent lung inflammation and immune-mediated pathology in RSV-infected mice (20-22). However, the role of Tregs during RSV infection in humans is currently unclear.

In this study, we observe a reduction in the frequency of activated Tregs (aTregs) in the peripheral blood of RSV-infected infants compared with controls. We also find that resting Treg (rTreg) frequencies decrease as RSV disease progresses. To understand the relationship between Treg expression and respiratory cytokine levels we also measured a panel of cytokines in the nasal secretions of each infant. We found that RSV-infected infants display an increased level of a wide range of proinflammatory cytokines. Importantly, we observed a reduction in interleukin (IL) -33, a cytokine required for Treg maintenance within mucosal tissues. Overall, our results demonstrate a

\footnotetext{
'Department of Microbiology, University of lowa, lowa City, lowa; ${ }^{2}$ Department of Pediatrics, University of lowa, lowa City, lowa; ${ }^{3}$ nstitute for Clinical and Translational Science, University of lowa, lowa City, lowa; ${ }^{4}$ Department of Internal Medicine, University of lowa, lowa City, lowa; ${ }^{5}$ Interdisciplinary Graduate Program in Immunology, University of lowa, lowa City, lowa; ${ }^{\circ}$ Department of Pathology, University of lowa, lowa City, lowa. Correspondence: Steven Varga(steven-varga@uiowa.edu)

Received 7 April 2016; accepted 20 April 2016; advance online publication 3 August 2016. doi:10.1038/pr.2016.130
} 
reduction in Treg frequencies and an increase in inflammatory cytokines in the lung in RSV-infected infants indicating that a failure to properly regulate the host immune response may contribute to the severity of RSV-induced disease.

\section{RESULTS}

\section{Characteristics of the Study Population}

In this study, 40 infants presenting with respiratory infection were enrolled. PCR and cultures of respiratory secretions determined that $25(62.5 \%)$ of the infants were infected with RSV. Two of the RSV-infected infants exhibited coinfections, one with adenovirus and the other with influenza. The remaining infants were infected with human metapneumovirus $(10 \%)$, adenovirus $(7.5 \%)$, parainfluenza virus $(5 \%)$, influenza $(2.5 \%)$ or bacteria $(12.5 \%)$. As expected $(24,25)$, RSV was the most prevalent pathogen detected in infants hospitalized with respiratory infections.

Control samples were collected from infants admitted to the hospital for elective surgery with no report of respiratory illness. Sex, age at sample collection, and gestational age were not found to be significantly different between the controls and RSV-infected infants (Table 1 and data not shown). Patient history showed no difference between the controls and RSV-infected infants with regard to a family history of asthma, second hand exposure to tobacco smoke, previous respiratory infections, infants with siblings or household pets (Table 1 and data not shown). In addition, a small number of RSV-infected infants in the study were premature (gestation $<37 \mathrm{wk} ; n=4$ ). We found that gestational age may have altered the response to two cytokines measured (see Supplementary Table S1 online). Furthermore, a small portion of infants from both controls and the RSV-infected infants were administered palivizimab prior to hospitalization but no difference was observed between the

Table 1. Demographic and treatment data of study population

\begin{tabular}{lcc}
\hline & Control $(n=17)$ & RSV $(n=23)$ \\
\hline Male/female & $9 / 8$ & $14 / 9$ \\
Age $^{\text {a }}$ (range) & $1.7(0.1-8.7)$ & $3.3(0.4-10.1)$ \\
Gestational age $^{\text {a }}$ (range) & $37.3(30-39)$ & $36.8(28-39)$ \\
Family history of asthma & $13 \%$ & $22 \%$ \\
Household smokers & $13 \%$ & $22 \%$ \\
Previous respiratory infection & $7 \%$ & $13 \%$ \\
Siblings & $67 \%$ & $86 \%$ \\
Household pets & $38 \%$ & $13 \%$ \\
Palivizumab & $6 \%$ & $9 \%$ \\
Oxygen received & $\mathrm{NA}$ & $91 \%$ \\
Use of CPAP & $\mathrm{NA}$ & $35 \%$ \\
Use of heliox & $\mathrm{NA}$ & $0 \%$ \\
Mechanical ventilation & $\mathrm{NA}$ & $22 \%$ \\
Steroids & $\mathrm{NA}$ & $13 \%$ \\
Albuterol & $\mathrm{NA}$ & $35 \%$ \\
\hline
\end{tabular}

CPAP, continuous positive airway pressure; $\mathrm{NA}$, not applicable; RSV, respiratory syncytial virus.

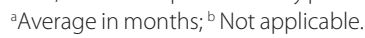

control and RSV-infected infants (Table 1). Overall, no significant difference in the characteristics of the study populations was observed.

Infants received supportive care based on their severity of illness (Table 1). However, no intervention was associated with a significant change in either the frequency of $\mathrm{T}$ cell populations or cytokine concentrations (data not shown).

\section{aTregs are Reduced in RSV-infected Infants}

Human Tregs can be divided into two distinct subsets. rTreg are defined by high expression of CD45RA and intermediate expression of either Foxp3 or CD25. aTreg are defined by low expression of CD45RA and high expression of either Foxp3 or CD25 as previously reported (26). Using a similar gating strategy (Figure 1a and see Supplementary Figure S1 online), the frequency of rTregs in the peripheral blood did not exhibit a significant difference between RSV-infected infants and controls (Figure 1b). However, we observed a reduced frequency of aTregs in the peripheral blood of RSV-infected infants compared with age-matched controls (Figure 1c). Furthermore, the frequencies of conventional naive and memory CD4 $\mathrm{T}$ cell populations were not altered in RSV-infected infants (Figure 1d,e). The frequency of proliferating CD4 T cells, as measured by Ki-67 expression, did not significantly differ between RSV-infected infants and controls (Figure 1f). Thus, while aTreg frequencies were reduced in RSV-infected infants, the proportion of proliferating aTregs was similar. Overall, RSV-infected infants displayed a reduced frequency of aTregs in the peripheral blood, with no perturbation observed in any other $\mathrm{CD} 4 \mathrm{~T}$ cell subset examined.

\section{Cytokine Concentrations Increase in RSV-Infected Infants}

To understand the relationship between Treg expression and respiratory cytokine levels we also measured a panel of 24 cytokines and chemokines. Of those cytokines analyzed, eight were observed at significantly greater levels in nasal excretions of RSV-infected infants compared with controls (Table 2). The Th1-associated cytokines interferon (IFN)- $\gamma$ and tumor necrosis factor (TNF)- $\alpha$ were significantly increased in RSVinfected infants (Table 2). The Th2-associated cytokine IL-4 was also significantly increased in RSV-infected infants, however, the Th2-associated cytokine IL- 5 was undetectable and IL-13 showed no significant difference between groups (Table 2). Furthermore, the proinflammatory and pyrogenic cytokines IL- $1 \alpha$ and IL- 6 were also increased in RSV-infected infants. IL-1 $\beta$ exhibited the greatest increase in RSV-infected infants indicating inflammasome activation (Table 2). Only IL-33, a danger signal induced in response to tissue damage (27), was significantly reduced in RSV-infected infants $(P=$ 0.0561, Table 2). Six cytokines and chemokines, including IL-5, IL-9, IL-17F, IL-17E, Eotaxin, and TSLP were undetectable in both RSV-infected infants and controls. Overall, our data demonstrates an increase in Th1, Th2 and proinflammatory cytokines while IL-33 is decreased in RSV-infected infants compared with controls. 


\section{Articles | Christiansen et al.}

a

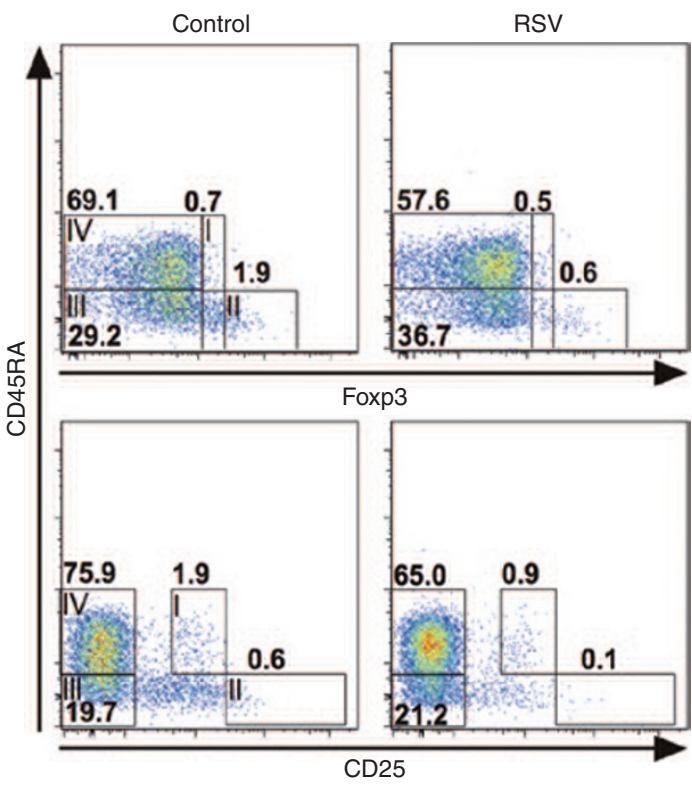

b
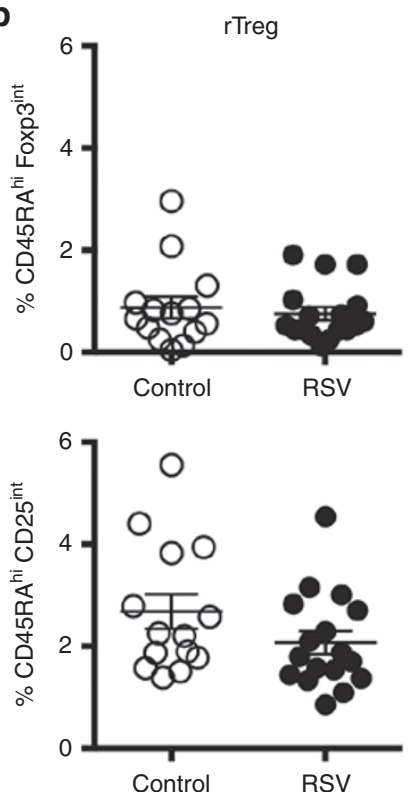

C
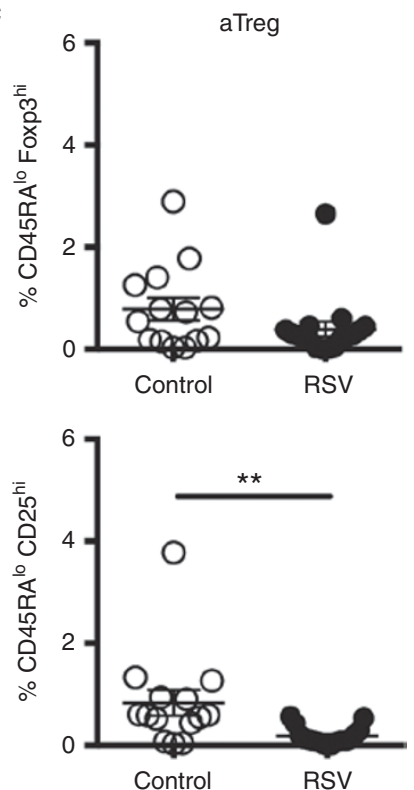

d

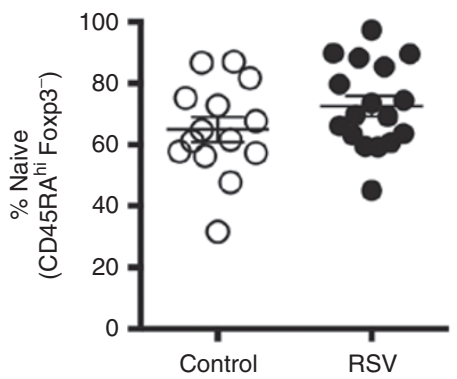

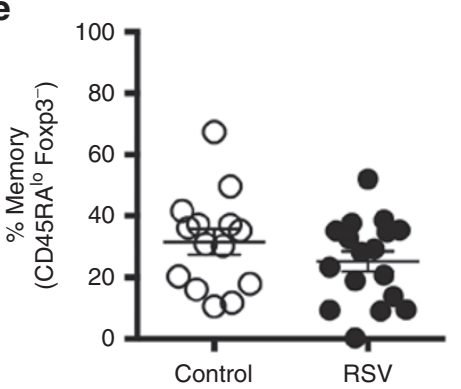

f

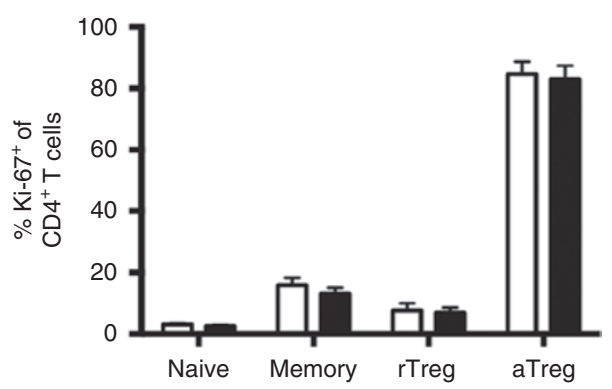

Figure 1. aTreg frequencies are reduced in the peripheral blood of RSV-infected infants. PBMCs from RSV-infected infants and controls were stained to

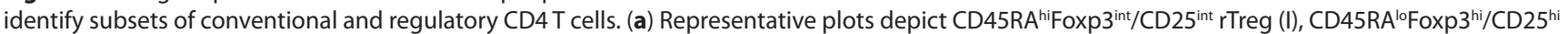
aTreg (II), CD45RA ${ }^{\text {hiFoxp3 }}$ naive conventional CD4 T cells (III), and CD45RA ${ }^{\text {loFoxp3 }}$ memory conventional CD4 T cells (IV) from control and RSV-infected infants. The cumulative frequencies of resting (b) and activated (c) Tregs are shown for controls and RSV-infected infants by two staining methods. (d) Naive and (e) memory conventional CD4 T cell frequencies are shown from all groups. (f) Ki-67 expression from each CD4 T cell subset defined by CD45RA and Foxp3 expression is shown for both control (white bars) and RSV-infected infants (black bars). All samples are gated on $C D 4^{+} C D 3^{+} T$ cells. Data represents the mean \pm SEM for both controls $(n=13)$ and RSV $(n=17)$ groups. ${ }^{* *} P<0.01$. aTregs, activated Tregs; RSV, respiratory syncytial virus; SEM, standard error of mean; rTreg, resting Treg; PBMCs, peripheral blood mononuclear cells.

\section{Resting Treg Frequencies Decrease as RSV Symptoms Progress} Upon TCR activation, rTregs upregulate Foxp3 expression and convert to aTregs (26). Thus, if Tregs are activated following RSV infection, we would expect to observe a decrease in rTregs and an increase in aTregs as RSV infection progressed. Therefore, we examined the change in the rTreg and aTreg population over the course of an acute respiratory infection in infants. A significant decline in the rTreg frequency was observed in the blood of RSV-infected infants as the days postsymptom onset increased (Figure 2). Thus, rTreg prevalence in the blood decreased as RSV disease progressed. However, we did not see an overall reduction in rTregs potentially because many of the samples were collected at an early time point following the initial onset of symptoms. In addition, we did not observe a subsequent increase in aTregs. This suggests that while the decline in rTregs may be due to conversion to aTregs, the aTregs were either migrating from the blood or undergoing apoptosis during RSV infection.

\section{Th17-associated Cytokines are Associated with Reduced Respiratory Signs and Symptoms}

The impact of IL-17 on disease severity following RSV infection has been controversial. Two previous studies have found infants with moderate RSV infection expressed more IL-17 than those with severe infection $(28,29)$. However, IL-17 was found to be pathogenic in the murine model of RSV resulting in enhanced mucus production and neutrophilic infiltrate (18). Here we found that Th17-associated cytokines IL-1 $\beta$, IL-17A, and IL-23 were all elevated when either difficulty breathing or retraction was not reported by the infant's doctor following examination (Figure 3). These results were found following extensive analysis to determine associations between multiple disease parameters and cytokine levels (see Supplementary Table S2 online). No association was observed for either the Th1-associated cytokine TNF- $\alpha$ or the Th2-associated cytokine IL-13 when either difficulty breathing or retraction was reported in the RSV-infected infants 
Table 2. Cytokines and chemokines in nasopharyngeal aspirates (pg/ml)

\begin{tabular}{|c|c|c|c|c|c|c|c|c|c|c|c|}
\hline & \multicolumn{4}{|c|}{ Control $(n=17)$} & \multicolumn{4}{|c|}{$\operatorname{RSV}(n=23)$} & \multirow[b]{2}{*}{ LOD } & \multirow[b]{2}{*}{$P$} & \multirow[b]{2}{*}{ Sig } \\
\hline & Avg & Median & $75^{\text {th }} \mathrm{Q}$ & $25^{\text {th }} \mathrm{Q}$ & Avg & Median & $75^{\text {th }} \mathrm{Q}$ & $25^{\text {th }} \mathrm{Q}$ & & & \\
\hline IL-1 $\alpha$ & 12.06 & 3.65 & 10.26 & 3.65 & 38.76 & 15.6 & 31.60 & 5.52 & 3.65 & 0.0509 & $\dagger$ \\
\hline IL-4 & 4.30 & 3.87 & 3.87 & 3.87 & 7.13 & 3.87 & 5.26 & 3.87 & 3.87 & 0.0317 & * \\
\hline IL-6 & 33.26 & 3.64 & 15.60 & 3.08 & 66.94 & 37.63 & 74.05 & 18.43 & 0.45 & 0.0694 & $\dagger$ \\
\hline IL-7 & 2.44 & 2.38 & 2.86 & 1.63 & 3.04 & 2.97 & 4.10 & 2.43 & 0.51 & 0.6553 & \\
\hline IL-12p40 & 4.94 & 3.69 & 3.69 & 3.69 & 7.38 & 4.15 & 6.67 & 3.69 & 3.69 & 0.1300 & \\
\hline IL-12p70 & 0.84 & 0.62 & 0.86 & 0.62 & 1.08 & 0.62 & 0.92 & 0.62 & 0.62 & 0.4336 & \\
\hline IL-13 & 2.40 & 2.32 & 3.13 & 1.83 & 3.08 & 3.155 & 4.41 & 1.82 & 0.57 & 0.4330 & \\
\hline IL-15 & 0.81 & 0.61 & 0.61 & 0.61 & 1.70 & 0.98 & 2.04 & 0.61 & 0.61 & 0.2768 & \\
\hline IL-17A & 0.75 & 0.64 & 0.64 & 0.64 & 0.95 & 0.64 & 0.91 & 0.64 & 0.64 & 0.7413 & \\
\hline TNF- $\alpha$ & 4.21 & 0.65 & 2.23 & 0.65 & 17.41 & 7.98 & 33.88 & 1.86 & 0.65 & 0.0858 & $\dagger$ \\
\hline IFN- $\gamma$ & 0.67 & 0.59 & 0.59 & 0.59 & 2.85 & 0.59 & 1.23 & 0.59 & 0.59 & 0.0002 & $\neq$ \\
\hline MDC & 26.94 & 27.33 & 35.86 & 15.60 & 29.52 & 24.42 & 40.94 & 18.82 & 15.6 & 0.6737 & \\
\hline RANTES & 19.88 & 3.85 & 3.85 & 3.85 & 56.08 & 23.51 & 70.41 & 12.42 & 3.85 & 0.0001 & $\neq$ \\
\hline
\end{tabular}

Numbers in bold denote those values that were significantly different from the controls.

IFN, interferon; IL, interluekin; MDC, macrophage-derived chemokine; RANTES, regulated on activation, normal T cell expressed and secreted; RSV, respiratory syncytial virus; Avg, average; $Q$, quartile; $L O D$, limit of detection; $P,=P$-value as determined by two-tailed $t$-test; Sig, significance.

$\dagger \leq 0.1,{ }^{*} \leq 0.05,{ }^{* *} \leq 0.01$, and ${ }^{\ddagger} \leq 0.001$.

a

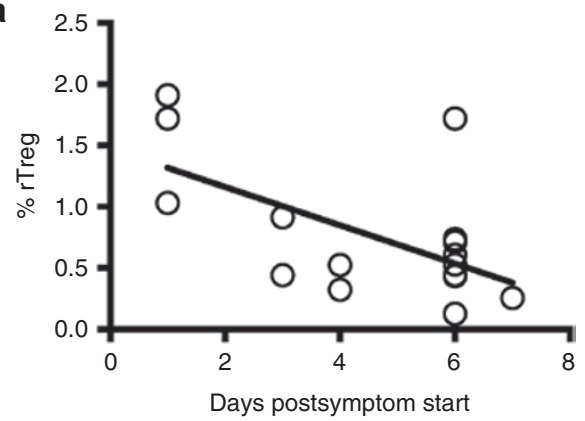

b

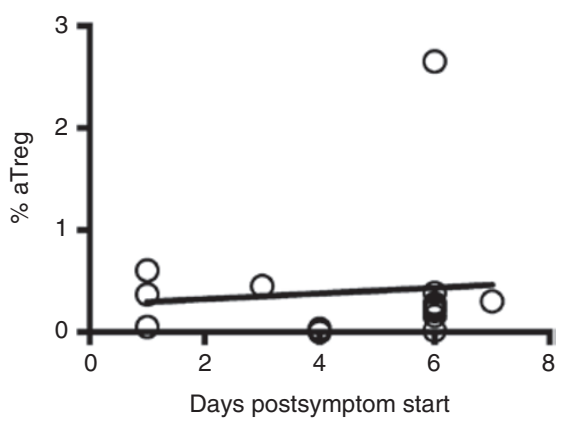

Figure 2. rTreg frequencies decrease as RSV disease progresses. The coefficients of determination between CD45RA $A^{\text {hiF }}$ oxp $3^{\text {int }}$ rTregs $\left(\mathbf{a}, R^{2}=\right.$ $0.3569)$ or CD45RA ${ }^{\text {lo Foxp }} 3^{\text {hi }}$ aTregs $\left(b, R^{2}=0.0089\right)$ and onset of symptoms in RSV-infected infants were calculated along with the $P$-values measuring significance of association $(\mathbf{a}, P=0.0113 ; \mathbf{b}, P=0.7187)$. aTregs, activated Tregs; RSV, respiratory syncytial virus; rTreg, resting Treg.
(Figure 3 and see Supplementary Table S2 online). Moreover, no cytokines were associated with any medical treatment that the children received (data not shown). Thus, Th17-associated cytokines may not be mediators of lung disease during infant RSV infection.

\section{DISCUSSION}

Respiratory syncytial virus infection has been shown to alter the inflammatory milieu of infected infants $(13,16)$, however, regulation of this inflammatory response has not been well studied. Here, we examined both the cytokine milieu and the expression of regulatory and conventional CD4 T cells in infants hospitalized with RSV infection compared with uninfected controls. We found that RSV infection in infants induces a significant reduction in aTregs but not rTregs compared with controls. These findings are similar to a recent study performed by Raiden et al. in Argentina (30). However, Raiden et al. also observed a reduction in all Foxp3 expressing CD4 $\mathrm{T}$ cells irrespective of subset (30). The differences in responsiveness of the rTreg and activated CD4 $\mathrm{T}$ cell subsets observed between the studies may be due to differences in circulating RSV strains or in the timing of sample collection after infection. However, in agreement with our results, Raiden et al also observed a reduction in the frequency of aTregs in the blood that may be the result of aTreg migration from the blood to the site of the infection in the lung. Alternatively, aTreg numbers 


\section{Articles | Christiaansen et al.}
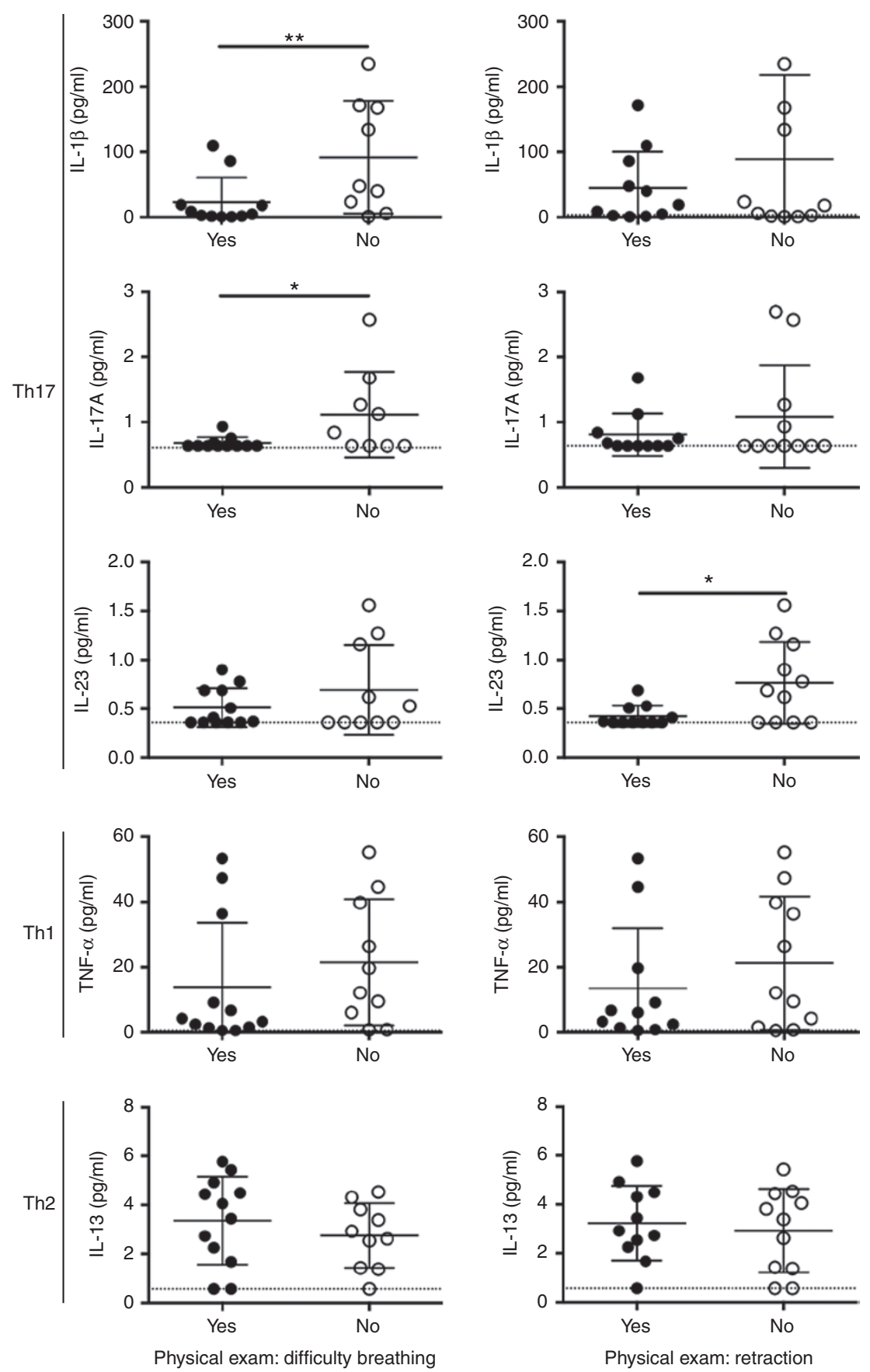

Figure 3. Th17-associated cytokines correlate with reduced difficulty breathing and retraction. Physical exam findings of difficulty breathing (left) and retraction (right) are associated with Th17-associated but not Th1- or Th2-associated cytokine concentrations in nasal excretions from RSV-infected infants. Data represent the mean \pm SEM for both controls $(n=13)$ and RSV $(n=17)$ groups. ${ }^{*} P<0.05 .{ }^{* *} P<0.01$. RSV, respiratory syncytial virus; SEM, standard error of mean; Th1, T helper1.

may be reduced in all peripheral tissues following RSV infection. We also observe that rTreg frequencies in the peripheral blood are reduced as RSV disease progresses. rTregs are believed to represent a long-lived Treg population that upon activation proliferate and convert to aTregs (26). Thus, a reduction in rTregs should result in an increase in aTregs, however, we did not observe this in our cohort of RSV-infected infants. Consequently, RSV infection appears to drive Treg activation as a result of which Tregs either leave the blood to enter the lung or undergo apoptosis. Further understanding the fate of Tregs is vital to understanding the regulation of the RSV immune response in infants. This reduction of Tregs in the blood is not observed in the murine model of RSV (20). In mice, Treg frequencies show a slight increase in the blood and are greatly expanded in the lung following RSV infection (20). Tregs have been shown to be vital in limiting the lung immunopathology observed in mice following RSV infection by regulating $\mathrm{T}$ cell recruitment, activation and cytokine production 
(20-22,31). It appears that Tregs do not follow a similar distribution pattern in RSV-infected infants and thus may not be present in the lung in sufficient numbers to properly regulate the host immune response.

To understand the correlation between Treg subset distribution and respiratory cytokine levels we also measured a panel of cytokines in the same RSV-infected infants and controls. We found that RSV-infected infants exhibited an increase in both Th1- and Th2-associated cytokines, though both are expressed at very low levels. A previous study with nasal samples from over $800 \mathrm{RSV}$-infected children below 5 y of age also observed an increase in the Th1-associated cytokine TNF- $\alpha$, but did not observe any detectable levels of Th2 cytokines (14), however, their assay may have had a higher limit of detection. Nevertheless, it is difficult to determine if the low levels of the Th1- and Th2-associated cytokines we observed are sufficient to impact RSV disease severity. Furthermore, Tregs are known to control both Th1- and Th2-associated cytokine responses in mice, however, no correlation was observed between the level of these cytokines and the frequency of either aTregs or rTregs within each individual (data not shown).

While the majority of cytokines measured were increased following RSV infection, IL-33 was found to be reduced in RSV-infected infants compared with controls. This is in contrast to recent studies in which IL-33 was observed to increase in mice and human infants following RSV infection (32-34). However, human RSV strain differences and severity of disease may explain the observed differences in IL-33 levels in infants. The RSV infected infants in our study exhibited severe RSV infection requiring hospitalization and intensive care whereas the infants enrolled in the study by Saravia et al. only required clinical presentation of RSV symptoms (32), thus increased disease severity may result in reduced IL-33 levels. IL-33 is believed to play an important role in RSV infection through its role as an alarmin that serves as a warning of tissue damage to the host (35-38), and thus may play an important role in activating the immune response. Furthermore, IL-33 has been shown to be necessary for Treg accumulation and maintenance in the mucosal site of the intestines (39). IL-33 may play a similar role for Treg recruitment and maintenance in other mucosal sites such as the lung. Importantly, our observed reduction in IL-33 levels could explain the reduction in the frequency of aTregs observed in the RSV-infected infants. Furthermore, the reduction in IL-33 from basal levels may indicate apoptotic rather than necrotic death of endothelial cells which has been shown to reduce IL-33 levels (40). The observed reduction in IL-33 may play an important role in explaining the severity of disease observed in these RSV-infected infants. However, our observations were made in two separate compartments, with IL-33 in the nasal washes and Tregs in the blood. Further, studies of Tregs in human airways will help to determine the connection between IL-33 and Tregs during RSV infection.

While Th17-associated cytokines were not found to change between RSV-infected infants and controls, observed symptoms within the RSV-infected infants were associated with the presence of Th17-associated cytokines. Each of the
RSV-infected infants presented with one or more respiratory symptoms including wheezing, retraction, congestion, and difficulty breathing. Extensive analysis of presented disease parameters identified a reduction in Th17-associated cytokines but not Th1- or Th2-ssociated cytokines when difficulty breathing and retraction were observed. Two previous studies reported similar findings where infants with moderate RSV infection expressed more IL-17 than those with severe infection $(28,41)$. However, IL-17 was found to be pathogenic in the murine model of RSV resulting in enhanced mucus production and neutrophilic infiltrate (18). While they found that IL-17 mediated enhanced disease, they also observed enhanced viral clearance in RSV-infected mice (18). IL-17 appears to be induced by RSV infection in both mice and humans, however, IL-17 appears to play a protective role by an unknown mechanism in human RSV infection.

Our results indicate that aTregs decrease in the peripheral blood following severe RSV infection in infants. This reduced regulation is associated with increased Th1-, Th2-, and inflammasome-associated cytokines. These results together with a reduction in the alarmin cytokine IL-33 indicate a lack of regulation resulting in an unbalanced immune response following RSV infection in infants. Understanding how the virus modulates the host inflammatory response can help to determine what immune targets are beneficial in treatment and prevention of RSV-infection in infants.

\section{METHODS \\ Study Population}

The study population was comprised of infants below $1 \mathrm{y}$ of age hospitalized at the University of Iowa Hospitals and Clinics between January 2013 and June 2014 that presented with suspected viral respiratory illness. Viral infection was confirmed by PCR of nasopharyngeal secretions. Infants $<1$ y of age admitted to the hospital without respiratory symptoms were included as controls. All samples were obtained within $48 \mathrm{~h}$ of hospital admission. Demographic information and family history were obtained from the parents. Physical examination and treatment information was obtained from medical records and the primary medical team. Children were excluded if they had a history of immunodeficiency, congenital cardiac disease, hemodynamic instability, deemed at risk of death, or lacked parental consent. The study was approved by the University of Iowa Human Subjects Office (IRB-01 201202786) and written consent was obtained from all of the patients' guardians.

\section{Nasal Fluid Cytokine Determination}

Nasal washes and tracheal aspirates were treated with an equal volume of $0.1 \%$ TritonX PBS, $1 \mu \mathrm{l} / \mathrm{ml}$ protease inhibitor cocktail (P8340; Sigma-Aldrich, St. Louis, MO) and frozen at $-80^{\circ} \mathrm{C}$. Cytokine concentrations were measured using a multiplex assay per the manufacturer's instructions (EMD Millipore, Billerica, MA).

\section{Peripheral Blood Mononuclear Cell Isolation}

Peripheral blood mononuclear cells (PBMCs) were isolated from $2 \mathrm{ml}$ of blood collected in $4 \mathrm{ml} \mathrm{Na}$ Heparin green top tubes (BD, Franklin Lakes, NJ). PBMCs were isolated using Ficoll-Paque Plus (GE Healthcare Life Sciences, Pittsburgh, PA). Cells were resuspended at $2 \times 10^{6}$ cells $/ \mathrm{ml}$ in $10 \%$ dimethylsulfoxide fetal calf serum and frozen at $-80^{\circ} \mathrm{C}$ followed by long-term storage in liquid nitrogen.

\section{Flow Cytometry}

PBMCs were thawed and stained with anti-CD25 (PE, BC96) and anti-CD45RA (eFlour450, HI100) extracellularly. Cells were fixed using Foxp3 Fixation Buffer and stained in permeabilization buffer 


\section{Articles $\mid$ Christiaansen et al.}

with antibodies specific to CD3 (Biotin, OKT3; Qdot 605, Invitrogen, Grand Island, NY), CD4 (Percp-efluor710, SK3), Foxp3 (PE-Cy7, 236A/E7; all antibodies were obtained from eBioscience, San Diego, $\mathrm{CA}$ ) and Ki-67 (Fluorescein isothiocyanate, B65; BD Bioscience, San Jose, CA). Samples were run on a BD LSR Fortessa (BD Biosciences) and the data was analyzed using FlowJo software version 9.9.2 (Tree Star, Ashland, OR).

\section{Statistical Analysis}

Statistical analyses were performed using GraphPad Prism software version 6.0h (La Jolla, CA) to compare T cell frequencies between control and RSV patients. Groups were compared using the MannWhitney test for unpaired samples. Additional statistical analyses were performed using SAS (SAS Institute, Cary, NC). Pearson correlations were calculated to determine associations between $\mathrm{T}$ cell populations, cytokine concentrations, and patient data (disease level, days of post-symptoms, and age). A linear modeling framework was used to determine associations between predictors (patient survey information and physical exam results) and outcomes ( $\mathrm{T}$ cell populations and cytokine concentration).

\section{SUPPLEMENTARY MATERIAL}

Supplementary material is linked to the online version of the paper at http:// www.nature.com/pr

\section{STATEMENT OF FINANCIAL SUPPORT}

This study was supported by National Institute of Health (Bethesda, MD) grant U01 HL102288 (to L.D.) and the Children's Miracle Network and the Division of Pediatric Critical Care at the University of lowa (to M.A.S.)

Disclosure: The authors would like to note that there are no conflicts of interest to declare.

\section{REFERENCES}

1. Nair H, Nokes DJ, Gessner BD, et al. Global burden of acute lower respiratory infections due to respiratory syncytial virus in young children: a systematic review and meta-analysis. Lancet 2010;375:1545-55.

2. Glezen WP, Taber LH, Frank AL, Kasel JA. Risk of primary infection and reinfection with respiratory syncytial virus. Am J Dis Child 1986;140: 543-6.

3. García CG, Bhore R, Soriano-Fallas A, et al. Risk factors in children hospitalized with RSV bronchiolitis versus non-RSV bronchiolitis. Pediatr 2010;126:e1453-60.

4. Rivera CA, Gomez RS, Diaz RA, et al. Novel therapies and vaccines against the human respiratory syncytial virus. Expert Opin Investig Drugs 2015;24:1613-30.

5. Johnson S, Oliver C, Prince GA, et al. Development of a humanized monoclonal antibody (MEDI-493) with potent in vitro and in vivo activity against respiratory syncytial virus. J Infect Dis 1997;176:1215-24.

6. American Academy of Pediatrics Committee on Infectious Diseases; American Academy of Pediatrics Bronchiolitis Guidelines Committee Updated guidance for palivizumab prophylaxis among infants and young children at increased risk of hospitalization for respiratory syncytial virus infection. Pediatr 2014;134:415-420.

7. Johnson JE, Gonzales RA, Olson SJ, Wright PF, Graham BS. The histopathology of fatal untreated human respiratory syncytial virus infection. Mod Pathol 2007;20:108-19.

8. Peebles RS Jr, Graham BS. Pathogenesis of respiratory syncytial virus infection in the murine model. Proc Am Thorac Soc 2005;2:110-5.

9. Graham BS, Bunton LA, Wright PF, Karzon DT. Role of T lymphocyte subsets in the pathogenesis of primary infection and rechallenge with respiratory syncytial virus in mice. J Clin Invest 1991;88:1026-33.

10. Everard ML, Swarbrick A, Wrightham M, et al. Analysis of cells obtained by bronchial lavage of infants with respiratory syncytial virus infection. Arch Dis Child 1994;71:428-32.

11. Heidema J, Lukens MV, van Maren WW, et al. CD8+ T cell responses in bronchoalveolar lavage fluid and peripheral blood mononuclear cells of infants with severe primary respiratory syncytial virus infections. J Immunol 2007;179:8410-7.
12. Garofalo RP, Patti J, Hintz KA, Hill V, Ogra PL, Welliver RC. Macrophage inflammatory protein-1alpha (not T helper type 2 cytokines) is associated with severe forms of respiratory syncytial virus bronchiolitis. J Infect Dis 2001;184:393-9.

13. Legg JP, Hussain IR, Warner JA, Johnston SL, Warner JO. Type 1 and type 2 cytokine imbalance in acute respiratory syncytial virus bronchiolitis. Am J Respir Crit Care Med 2003;168:633-9.

14. Tabarani CM, Bonville CA, Suryadevara M, et al. Novel inflammatory markers, clinical risk factors and virus type associated with severe respiratory syncytial virus infection. Pediatr Infect Dis J 2013;32:e437-42.

15. Mangodt TC, Van Herck MA, Nullens S, et al. The role of Th17 and Treg responses in the pathogenesis of RSV infection. Pediatr Res 2015;78: 483-91.

16. Christiaansen AF, Knudson CJ, Weiss KA, Varga SM. The CD4 T cell response to respiratory syncytial virus infection. Immunol Res 2014;59:109-17.

17. Tekkanat KK, Maassab HF, Cho DS, et al. IL-13-induced airway hyperreactivity during respiratory syncytial virus infection is STAT6 dependent. J Immunol 2001;166:3542-8.

18. Mukherjee S, Lindell DM, Berlin AA, et al. IL-17-induced pulmonary pathogenesis during respiratory viral infection and exacerbation of allergic disease. Am J Pathol 2011;179:248-58.

19. Weiss KA, Christiaansen AF, Fulton RB, Meyerholz DK, Varga SM. Multiple CD4+ T cell subsets produce immunomodulatory IL-10 during respiratory syncytial virus infection. J Immunol 2011;187:3145-54.

20. Fulton RB, Meyerholz DK, Varga SM. Foxp3+CD4 regulatory T cells limit pulmonary immunopathology by modulating the CD8 $\mathrm{T}$ cell response during respiratory syncytial virus infection. J Immunol 2010;185:2382-92.

21. Loebbermann J, Thornton H, Durant L, et al. Regulatory T cells expressing granzyme B play a critical role in controlling lung inflammation during acute viral infection. Mucosal Immunol 2012;5:161-72.

22. Ruckwardt TJ, Bonaparte KL, Nason MC, Graham BS. Regulatory T cells promote early influx of CD8+ T cells in the lungs of respiratory syncytial virus-infected mice and diminish immunodominance disparities. J Virol 2009;83:3019-28.

23. Christiaansen AF, Boggiatto PM, Varga SM. Limitations of Foxp3(+) Treg depletion following viral infection in DEREG mice. J Immunol Methods 2014;406:58-65.

24. Shay DK, Holman RC, Newman RD, Liu LL, Stout JW, Anderson LJ. Bronchiolitis-associated hospitalizations among US children, 1980-1996. JAMA 1999;282:1440-6.

25. Leader S, Kohlhase K. Respiratory syncytial virus-coded pediatric hospitalizations, 1997 to 1999. Pediatr Infect Dis J 2002;21:629-32.

26. Miyara M, Yoshioka Y, Kitoh A, et al. Functional delineation and differentiation dynamics of human CD4+ T cells expressing the FoxP3 transcription factor. Immunity 2009;30:899-911.

27. Cayrol C, Girard JP. The IL-1-like cytokine IL-33 is inactivated after maturation by caspase-1. Proc Natl Acad Sci USA 2009;106:9021-6.

28. Faber TE, Groen H, Welfing M, Jansen KJ, Bont LJ. Specific increase in local IL-17 production during recovery from primary RSV bronchiolitis. J Med Virol 2012;84:1084-8.

29. Larrañaga CL, Ampuero SL, Luchsinger VF, et al. Impaired immune response in severe human lower tract respiratory infection by respiratory syncytial virus. Pediatr Infect Dis J 2009;28:867-73.

30. Raiden S, Pandolfi J, Payasliàn F, et al. Depletion of circulating regulatory $\mathrm{T}$ cells during severe respiratory syncytial virus infection in young children. Am J Respir Crit Care Med 2014;189:865-8.

31. Durant LR, Makris S, Voorburg CM, Loebbermann J, Johansson C, Openshaw PJ. Regulatory T cells prevent Th2 immune responses and pulmonary eosinophilia during respiratory syncytial virus infection in mice. J Virol 2013;87:10946-54.

32. Saravia J, You D, Shrestha B, et al. Respiratory syncytial virus disease is mediated by age-variable IL-33. PLoS Pathog 2015;11:e1005217.

33. Liu J, Wu J, Qi F, et al. Natural helper cells contribute to pulmonary eosinophilia by producing IL-13 via IL-33/ST2 pathway in a murine model of respiratory syncytial virus infection. Int Immunopharmacol 2015;28: $337-43$. 
34. Zeng S, Wu J, Liu J, Qi F, Liu B. IL-33 Receptor (ST2) signalling is important for regulation of Th2-mediated airway inflammation in a murine model of acute respiratory syncytial virus infection. Scand J Immunol 2015;81:494-501.

35. Moussion C, Ortega N, Girard JP. The IL-1-like cytokine IL-33 is constitutively expressed in the nucleus of endothelial cells and epithelial cells in vivo: a novel 'alarmin'? PLoS One 2008;3:e3331.

36. Carriere V, Roussel L, Ortega N, et al. IL-33, the IL-1-like cytokine ligand for ST2 receptor, is a chromatin-associated nuclear factor in vivo. Proc Natl Acad Sci USA 2007;104:282-7.

37. Palmer G, Gabay C. Interleukin-33 biology with potential insights into human diseases. Nat Rev Rheumatol 2011;7:321-9.
38. Tjota MY, Williams JW, Lu T, et al. IL-33-dependent induction of allergic lung inflammation by FcyRIII signaling. J Clin Invest 2013;123:2287-97.

39. Schiering C, Krausgruber T, Chomka A, et al. The alarmin IL-33 promotes regulatory T-cell function in the intestine. Nature 2014; 513:564-8.

40. Lüthi AU, Cullen SP, McNeela EA, et al. Suppression of interleukin-33 bioactivity through proteolysis by apoptotic caspases. Immunity 2009; 31:84-98.

41. Larrañaga CL, Ampuero SL, Luchsinger VF, et al. Impaired immune response in severe human lower tract respiratory infection by respiratory syncytial virus. Pediatr Infect Dis J 2009;28: $867-73$. 